\title{
TTK contributes to tumor growth and metastasis of clear cell renal cell carcinoma by inducing cell proliferation and invasion
}

\author{
X. D. LIU' ${ }^{1}$, D. W. YAO 2 , F. XIN ${ }^{2, *}$ \\ ${ }^{1}$ Department of Nephrology, The Second People's Hospital of Lianyungang, Lianyungang, Jiangsu, China; ${ }^{2}$ Department of Urology, The Second \\ People's Hospital of Lianyungang, Lianyungang, Jiangsu, China \\ ${ }^{*}$ Correspondence: xinfeng1983911@163.com
}

Received February 7, 2019 / Accepted May 29, 2019

\begin{abstract}
The aim of this study was to determine the expression levels of TTK in clear cell renal cell carcinoma (ccRCC) tissues and its possible link with the clinical pathologic characteristics and the prognosis of patients suffering this disease, and to further explore the potential role of TTK in the progression of ccRCC. Immunohistochemical (IHC) assays were performed to detect the expression levels of TTK in 112 samples of ccRCC tissues and corresponding non-tumor tissues. According to the results of IHC assays, patients were divided into TTK high expression and low expression group. Clinical analysis related to the clinical features (age, gender, T stage), and the potential link between TTK expression levels and clinical features were analyzed. In addition, the effects of TTK on the proliferation and invasion of ccRCC cells were detected through colony formation assay and transwell assays, respectively. The possible effects of TTK on tumor growth and metastasis were measured in mice. We found a high expression level of TTK in human ccRCC tissues from patients who received surgical treatment. We also found its expression level was obviously associated with clinical characteristics, such as $\mathrm{T}$ stage $(\mathrm{p}=0.008)$ and lymphatic metastasis $(\mathrm{p}=0.023)$. We further confirmed that knockdown of TTK suppressed cell proliferation and invasion in 2 types of ccRCC cells, HTB-47 and CRL-1932 cells. Furthermore, TTK contributes to tumor growth and metastasis of ccRCC in mice. We found that TTK affected the progression of ccRCC and further mechanically confirmed it could be a novel therapeutic target for ccRCC treatment.
\end{abstract}

Key words: clear cell renal cell carcinoma (ccRCC), TTK, proliferation, invasion, metastasis, therapeutic target

Renal cell carcinoma (RCC) is a type of urinary system cancer with high morbidity and mortality [1]. In United States, there were more than 60, 000 new diagnosed RCC patients and approximately 15, 000 deaths in 2017 [2-4]. Clear cell renal cell carcinoma (ccRCC) is a highly aggressive renal malignant tumor accounting for nearly $80 \%$ of RCC [5]. Traditional treatments, such as surgical resection, radiation and chemotherapy, seems to be ineffective against this type of highly aggressive tumor [6]. Over decades, targeted therapy for ccRCC has shown great promise [7]. It has been earlier reported that VHL is considered a potential molecular target of ccRCC, however, more mutations have been subsequently identified, such as PBRM1, and these mutations in ccRCC has resulted in further research of their possible role as therapeutic targets for ccRCC $[8,9]$. However, to combat this disease, more effective therapeutic targets are badly needed.

TTK, also known as Mps1, is a conserved dual-specificity kinase that functions as a key guardian of spindle pole assembly, meiosis, ciliogenesis, DNA damage response and cytokinesis [10-12]. TTK is also an essential component of SAC and is involved in ensuring proper orientation of sister chromatids [13]. Previous studies demonstrated that knockdown of TTK led to the aberrations in chromosome counts [11]. Additionally, TTK expression is tightly associated with the progression of cell cycle and it is degraded by the ubiquitin-proteasome pathway [14]. Interestingly, the overexpression of human TTK results in the over duplication of centrosome [14]. In view of the multiple critical functions of TTK, it might be involved in the regulation of cancer progression.

In fact, numerous studies have proved the critical role of TTK in cancer progression and metastasis. TTK is reported highly expressed in a variety of tumor types such as gastric cancer, thyroid papillary carcinoma and lung cancer [15-17]. Additionally in breast cancer, TTK ablation induced cell apoptosis and aberrant mitosis, and high TTK expression was associated with tumor grades [18]. Another research 
demonstrated that high expression of TTK contributes to cell proliferation with higher aneuploidy by blocking SAC threshold in colon cancer [19]. Although TTK is involved in the progression of several cancers, its potential effects on renal cell carcinoma remain unclear.

In this study, we evaluated the possible role of TTK on the progression of clear cell renal cell carcinoma. We initially found the high expression of TTK in human ccRCC tissues and explored the connection between TTK expression and clinical pathological features of patients. Ablation of TTK suppressed cell proliferation or invasion of ccRCC both in vitro and in mice. According to the previous results, we therefore thought TTK as a novel molecular target for the treatment of ccRCC.

\section{Materials and methods}

Bioinformatic analysis. We used GEPIA (http:/gepia. cancer-pku.cn/) to collate and analyze (The Cancer Genome Atlas) TCGA data with a threshold of $\mathrm{p}<0.05$ and $\operatorname{LogFC}>1$ or $<-1$ for differential genes, and the median was used as the basis for dividing the patients into two groups for KaplanMeier survival analysis, The $95 \%$ confidence interval is marked with a dotted line.

Antibodies, primers and shRNA plasmids. Rabbit anti-TTK (for IHC assays, 1:200 dilution, for immunoblot assays, 1:1000 dilution, ab219068, Abcam, Cambridge, UK), mice anti- $\beta$-actin (1:1000 dilution, ab8226, Abcam, Cambridge, UK).

The quantitative PCR primer sequences of TTK were as follows: forward, 5'-CGCAGCTTTCTGTAGAAATGGA-3' and reverse, 5'-GAGCATCACTTAGCGGAACAC-3'; the quantitative PCR primer sequences of GAPDH were as follows: 5'-GGTGGTCTCCTCTGACTTCAACA-3' and reverse, 5'-GTTGCTGTAGCCAAATTCGTTGT-3'. Readyto-package AAV TTK shRNA plasmids were bought from the Addgene plc. The shRNA sequences targeted TTK were as follows: sense, 5'-AAATGAAGACCTTACTGATGAAC-3'.

Human tissue samples and analysis. The 112 human ccRCC tissues and adjacent normal tissues examined in this study were collected from patients receiving surgical treatment in our hospital. The clinical characteristics are precisely recorded and listed in Table 1.

To explore the possible link between TTK expression and clear cell renal cell carcinoma, immunohistochemical (IHC) assays were conducted. Briefly, sample sections were fixed with $4 \%$ paraformaldehyde for approximately 30 minutes and subsequently blocked with 2\% BSA for another 30 minutes. Slides were subsequently incubated with TTK antibodies at room temperature for 2 hours. Then the sections were incubated with biotinylated secondary antibody for 1.5 hours, and diaminobenzidine was used as a chromogen substrate.

According to the staining results, we found that TTK was mainly located in the nucleus of human ccRCC tissues. We performed a scoring method as follows. Briefly, the propor- tion of positive stained cells was graded as follows: $0=0 \%$ stained cells; 1 represented $1-25 \%$ stained cells; 2 indicated $26-60 \%$ stained cells; $3=61-100 \%$ stained cells. The staining intensity was evaluated on a score of 0 (negative), 1 (low), 2 (modest) and 3 (strong). TTK expression levels were detected based on the staining index: score of staining intensity $\times$ score of stained cells percentage. Staining index $<4$ was considered TTK low expression, while staining index $>4$ or $=4$ was thought TTK high expression.

Cell culture and transfection. The 2 types of human ccRCC cell lines, HTB-47 and CRL-1932, were purchased from ATCC. Both HTB-47 and CRL-1932 cells were incubated in RPMI1640 medium, respectively, supplemented with $10 \%$ fetal bovine serum (Gibco, CA, USA) in a $5 \% \mathrm{CO}_{2}$ incubator.

The TTK shRNA plasmids were transfected into HTB-47 and CRL-1932 cells using Lipofectamine 2000 (11668019, Invitrogen, Carlsbad, CA, USA). The stably depletion HTB-47 cells was screened by its shRNA infection and used for the animal assays.

Quantitative PCR assay. Trizol reagent (15596026, Invitrogen, CA, USA) was used to extract total RNAs from human ccRCC cells. Then the RNA was reverse-transcribed using reverse transcriptase (M1701, Promega, Wisconsin, USA). Furthermore, total RNA was subsequently reverse transcribed to produce cDNA by synthesis system. Quantitative PCR was performed using SYBR Ex Taq kit (638319, Takara, Japan) and the expression levels of TTK were normalized to the expression of GAPDH.

Immunoblot assays. Tumor cells or tissues of ccRCC cancer were lysed using cell lysis buffer. Then the total proteins were analyzed through SDS-PAGE. Subsequently the polyvi-

Table 1. Relationships of TTK and clinicopathological characteristics in 112 patients with clear cell renal cell carcinoma.

\begin{tabular}{|c|c|c|c|c|c|}
\hline \multirow{3}{*}{ Feature } & \multirow{3}{*}{$\begin{array}{c}\text { All } \\
n=112\end{array}$} & \multicolumn{2}{|c|}{$\begin{array}{c}\text { TTK } \\
\text { expression }\end{array}$} & \multirow{3}{*}{$\chi^{2}$} & \multirow{3}{*}{ p-value } \\
\hline & & Low & High & & \\
\hline & & $\mathrm{n}=49$ & $n=63$ & & \\
\hline Age (year) & & & & 0.885 & 0.347 \\
\hline$<50$ & 47 & 23 & 24 & & \\
\hline$\geq 50$ & 65 & 26 & 39 & & \\
\hline Gender & & & & 2.401 & 0.121 \\
\hline Male & 59 & 22 & 37 & & \\
\hline Female & 53 & 27 & 25 & & \\
\hline T stage & & & & 6.977 & $0.008^{*}$ \\
\hline $\mathrm{T}_{1-2}$ & 49 & 25 & 24 & & \\
\hline $\mathrm{T}_{3-4}$ & 63 & 24 & 39 & & \\
\hline Differentiation & & & & 3.411 & 0.065 \\
\hline Low & 50 & 25 & 25 & & \\
\hline High & 62 & 24 & 48 & & \\
\hline Lymphatic metastasis & & & & 5.182 & $0.023^{*}$ \\
\hline No & 69 & 36 & 33 & & \\
\hline Yes & 43 & 13 & 30 & & \\
\hline
\end{tabular}


nylidene fluoride (PVDF) membranes were blocked with 5\% milk in TBST and incubated with the primary antibodies for the detection of TTK and $\beta$-actin at room temperature for $2 \mathrm{~h}$. Membranes were washed with TBST for 4 times and then the PVDF membranes were incubated with HRP-conjugate secondary antibodies for 45 minutes. Blots were detected with an ECL kit.

Colony formation assay. Approximately 1000 HTB -47 and CRL-1932 cells were plated into a 6-well culture plate and transfected with control or TTK shRNA plasmids and cultured in a $37^{\circ} \mathrm{C}, 5 \% \mathrm{CO}_{2}$ incubator. After 14 days, cells were fixed with PFA at room temperature for 20 minutes and stained with $0.2 \%$ crystal violet buffer for another 30 minutes and then washed with PBS twice. Then the number of colonies was manually counted and compared.

Cell proliferation assay. Cells were seeded into 96-well with complete medium, each well contained with 5000 cells and $200 \mu \mathrm{l}$ medium. Take out a 96 -wells every $24 \mathrm{~h}$ to evaluated cell proliferation using CCK8 (96992, Sigma, Saint Louis, USA) assay according to the manufacturer's instructions. Simply, $20 \mu \mathrm{l}$ CCK8 was added into each well and cultured in cell incubator for 4 hours, and the absorbance was evaluated using microplate reader at $570 \mathrm{~nm}$ (Bio-rad, Hercules, USA).

Transwell assay. HTB-47 and CRL-1932 cells were transfected with control or TTK shRNA plasmids for $48 \mathrm{~h}$ and then re-suspended in serum-free medium. The upper chambers of filters with $8.0 \mu \mathrm{m}$ membrane pores were containing $20 \%$ matrigel and incubated at $37^{\circ} \mathrm{C}$ for 30 minutes. Approximately 105 cells in $150 \mu \mathrm{l}$ of medium were then plated into the upper chambers of the inserts and induced to migrate toward the bottom chambers containing complete medium. After $24 \mathrm{~h}$, cells in the top chamber were removed, and cells were fixed in $4 \%$ paraformaldehyde for 25 minutes and stained with $0.1 \%$ crystal violet buffer for 30 minutes. The cell number was manually counted.

Cell scratch assays. The interested cells were seeded in 6-well plates and grew into full confluence overnight. A sterile pipette tip was used to introduce a scratch in the middle of each well. Next, the growth medium was discarded and replaced with fresh medium. The rate of migration towards the center of the wound was ascertained at indicated time points.

Tumor growth assay. All animal assay processes were approved by our Institutional Animal Care and Use Committee (IACUC). Briefly, HTB-47 cells were stably transfected with control or TTK shRNA lentivirus. About $10^{6}$ control or TTK depletion cells were subcutaneously implanted into athymic nude mice. After 2 weeks, the volume of each tumor was measured every 3 days.

Tumor metastasis assay. HTB-47 cells were infected with control or TTK shRNA lentivirus. About $5 \times 10^{5}$ cells were injected into the tail vein of athymic nude mice. After 8 weeks, tumors were isolated from mice in each group, weighted and compared.
Statistics. GraphPad 5.0 software was used for statistical analysis. All results in this study represented as mean \pm SD. The analysis of link between clinical features and protein levels were performed by $\chi^{2}$ analysis. Student's t-test was used for statistical comparisons. ${ }^{*}$ indicates $\mathrm{p}<0.05$ and represents significance.

\section{Results}

Information analysis of TTK in ccRCC patients. We first analyzed the expression level of TTK in ccRCC patients using GEPIA, an online analytical database that analyzes cases from TCGA, and found that TTK expression was significantly increased in tumor tissue compared with normal renal tissue (Figure S1A). A total of 523 cases of TCGA patients were analyzed, and divided into high and low expression groups according to the median. Comparing with the lower expression cases, patients with higher level of TTK shared a significantly worse disease-free survival (Figure $\mathrm{S} 1 \mathrm{~B}, \mathrm{p}=0.016$ ). These results give a clue of a potential role of TTK in ccRCC.

TTK is highly expressed in human ccRCC tissues. To evaluate the potential role of TTK in the progression of clear cell renal cell carcinoma, the expression levels of TTK in ccRCC tissues of patients who received surgical resection were examined through IHC assays. Interestingly, we found that TTK was mainly located in the nucleus of ccRCC cells (Figure 1A). To further explore the effects of TTK on ccRCC, we compared the difference of TTK expression levels in tumor tissues and the adjacent tissues through IHC assays. Consistent with our expectations, tumor tissues showed relative high expression of TTK, compared with adjacent non-tumor tissues (Figures 1A, 1B).

TTK expression levels correlate with clinical features of patients who underwent ccRCC. A total of 112 tumor tissue samples from ccRCC patients who underwent surgical resection were manual divided into TTK low and high expression groups, according to the staining intensity (Figure 1A and Table 1). According to the staining results, 49 patients exhibited low TTK expression, whereas 63 showed TTK high expression (Table 1).

We next assessed the clinical significance of TTK in ccRCC patients. Clinical features such as patient age, gender and clinical stage were evaluated. No clinical correlation was detected in features including patient age and tumor grade between TTK low and high expression groups (Table 1). Interestingly, our results indicated that TTK expression levels were markedly associated with $\mathrm{T}$ stage $(\mathrm{p}=0.008)$ and lymphatic metastasis $(\mathrm{p}=0.023)$ in patients with $\mathrm{ccRCC}$ (Table 1). Therefore, we found that TTK expression levels were associated with the clinical characteristics of patients with ccRCC.

Knockdown of TTK suppressed cell proliferation and invasion of ccRCC in vitro. To investigate the potential molecular mechanism of TTK in the progression of ccRCC, the shRNA specifically targeting TTK was trans- 
A
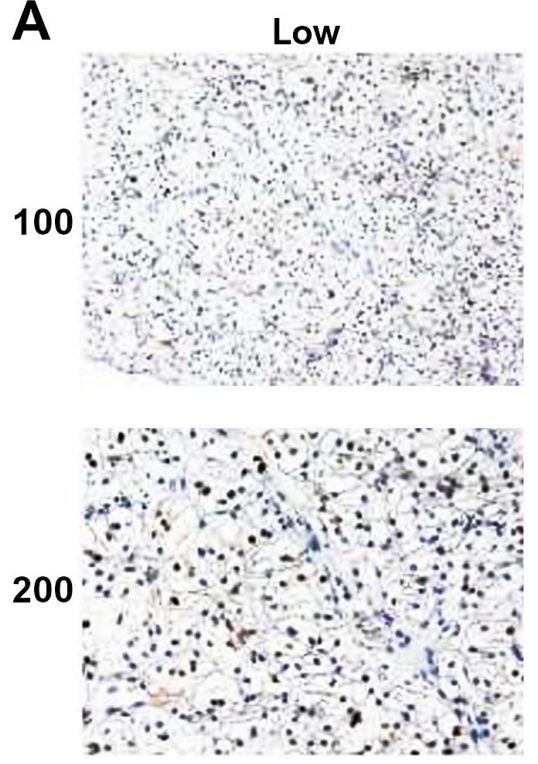

High
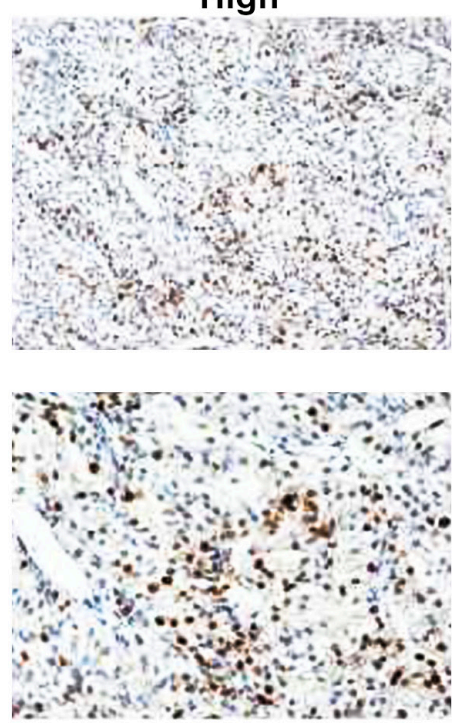

B

100

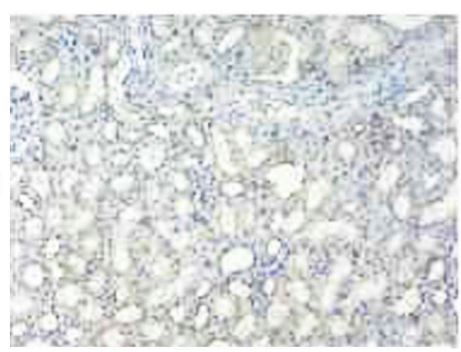

200

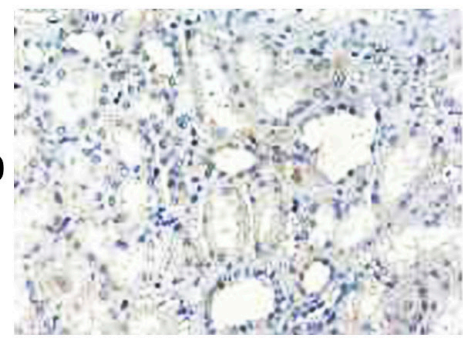

Figure 1. TTK was highly expressed in human ccRCC tissues. A) To detect TTK expression levels in human ccRCC tissues, IHC assays were performed, and the representative photographs were shown $(\times 100$ and $\times 200$ magnification, respectively). B) The results of IHC assays showed TTK expression levels in adjacent tissues $(\times 100$ and $\times 200$ magnification, respectively).

A

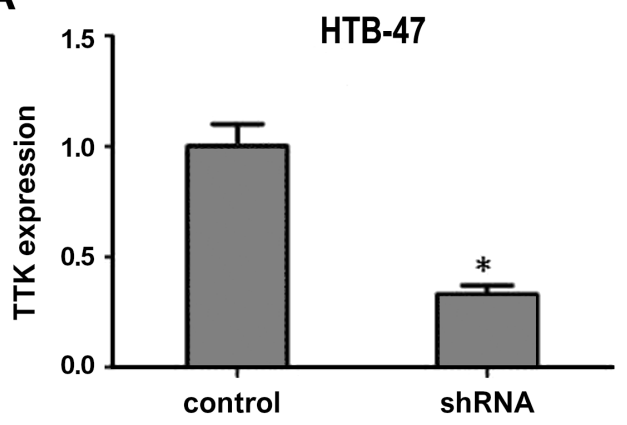

B

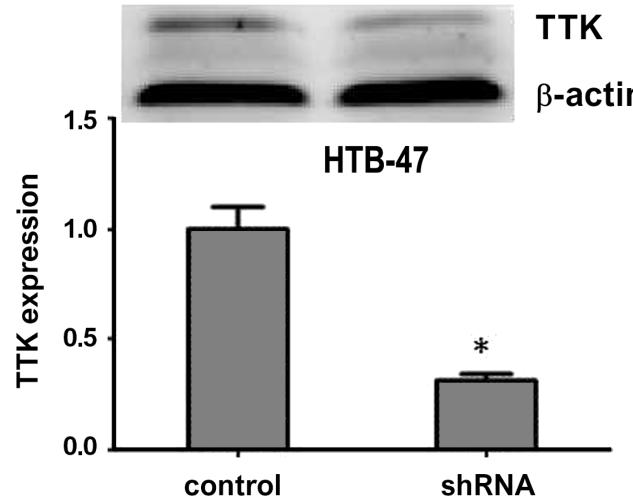

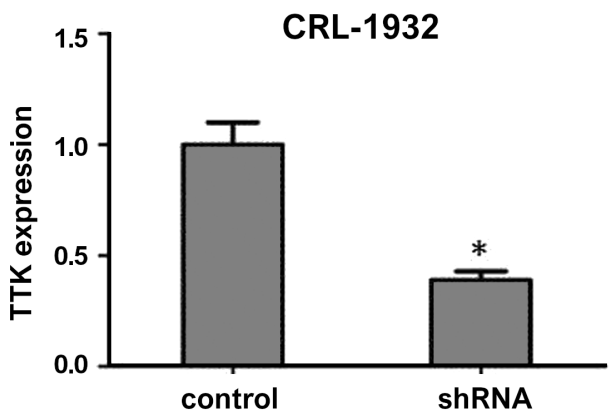

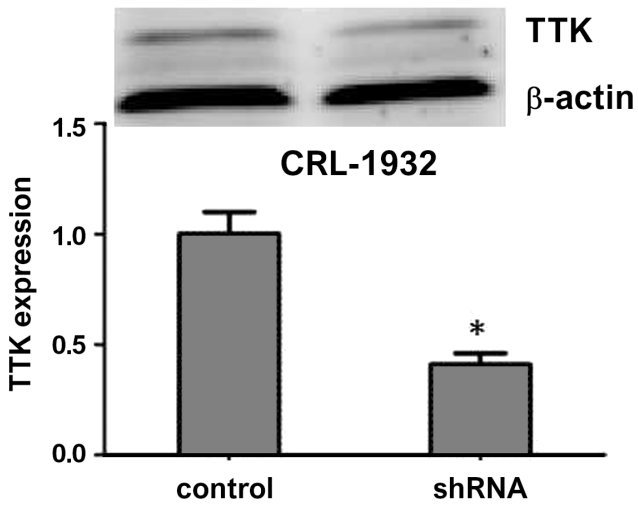

Figure 2. TTK expression levels were obviously reduced in both HTB-47 and CRL-1932 cells after the transfection of TTK shRNA. A) Quantitative PCR assays showed the dramatically decrease expression levels of TTK after the transfection of its shRNA plasmids in HTB-47 and CRL-1932 cells, respectively. B) Immunoblot assays revealed the efficiently decrease of TTK expression levels following the transfection of its shRNA in both HTB-47 and CRL-1932 cells. Results are presented as mean $\pm \mathrm{SD},{ }^{\star} \mathrm{p}<0.05$. 
fected into two types of human cells, HTB-47 and CRL-1932 cells, to suppress the expression of TTK. Quantitative PCR (Figure 2A) and western blot (Figure 2B) assays showed the shRNA of TTK effectively blocked its expression levels in both HTB-47 and CRL-1932 cells, respectively.

Subsequently, colony formation and CCK8 assays were conducted to assess the capacity of ccRCC cell proliferation. Results of manual count showed that TTK depletion reduced colony number or cell number (Figures $3 \mathrm{~A}$ and $3 \mathrm{~B}$ ).
We also evaluate the role of TTK on the invasion of HTB-47 and CRL-1932 cells though transwell and scratch assays. We found the depletion of TTK markedly suppressed the invasion capacity of both HTB-47 and CRL-1932 cells through membranes, which was reflected by a significant decrease of cell number (Figure 3C) We also found the depletion of TTK markedly suppressed the migration capacity of both HTB-47 and CRL-1932 cells, which was reflected by the significant bigger scratch space, respectively (Figure 3D).
A

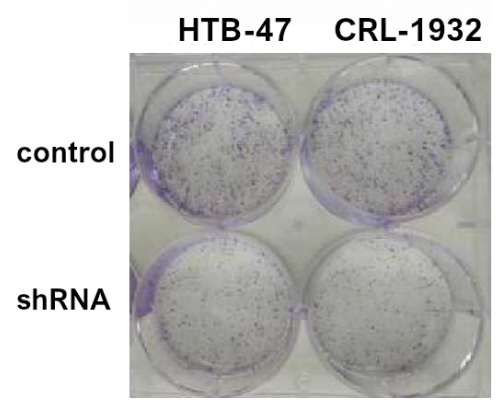

Figure 3. TTK contributes to tumor cell proliferation and invasion of ccRCC in vitro. A) Colony formation assays were performed using HTB-47 and CRL-1932 cells transfected with control or TTK shRNA plasmids, and the number of colony was manual counted. B) Detecting cell proliferation using CCK8 assay in HTB-47 and CRL-1932 cell lines transfected with control or TTK shRNA plasmids. C) Transwell assays using both HTB-47 and CRL-1932 cells transfected with control or TTK shRNA plasmids, and the capacity of invasion was quantified and compared according to cell number. D) Cell scratch assays. Results showed comparing with control, the TTK silencing cells showed bigger scratch space in HTB-47 and CRL-1932 cell lines, respectively $(\mathrm{p}<0.05)$. Results are presented as mean $\pm \mathrm{SD},{ }^{\star} \mathrm{p}<0.05$.
B

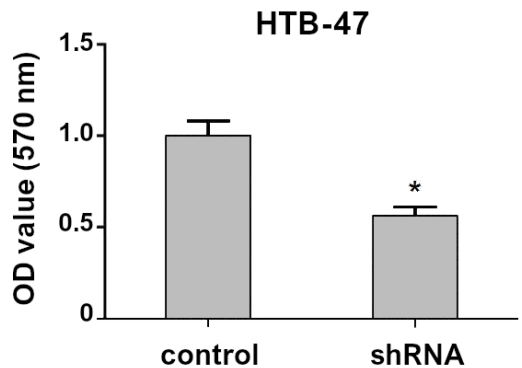

C
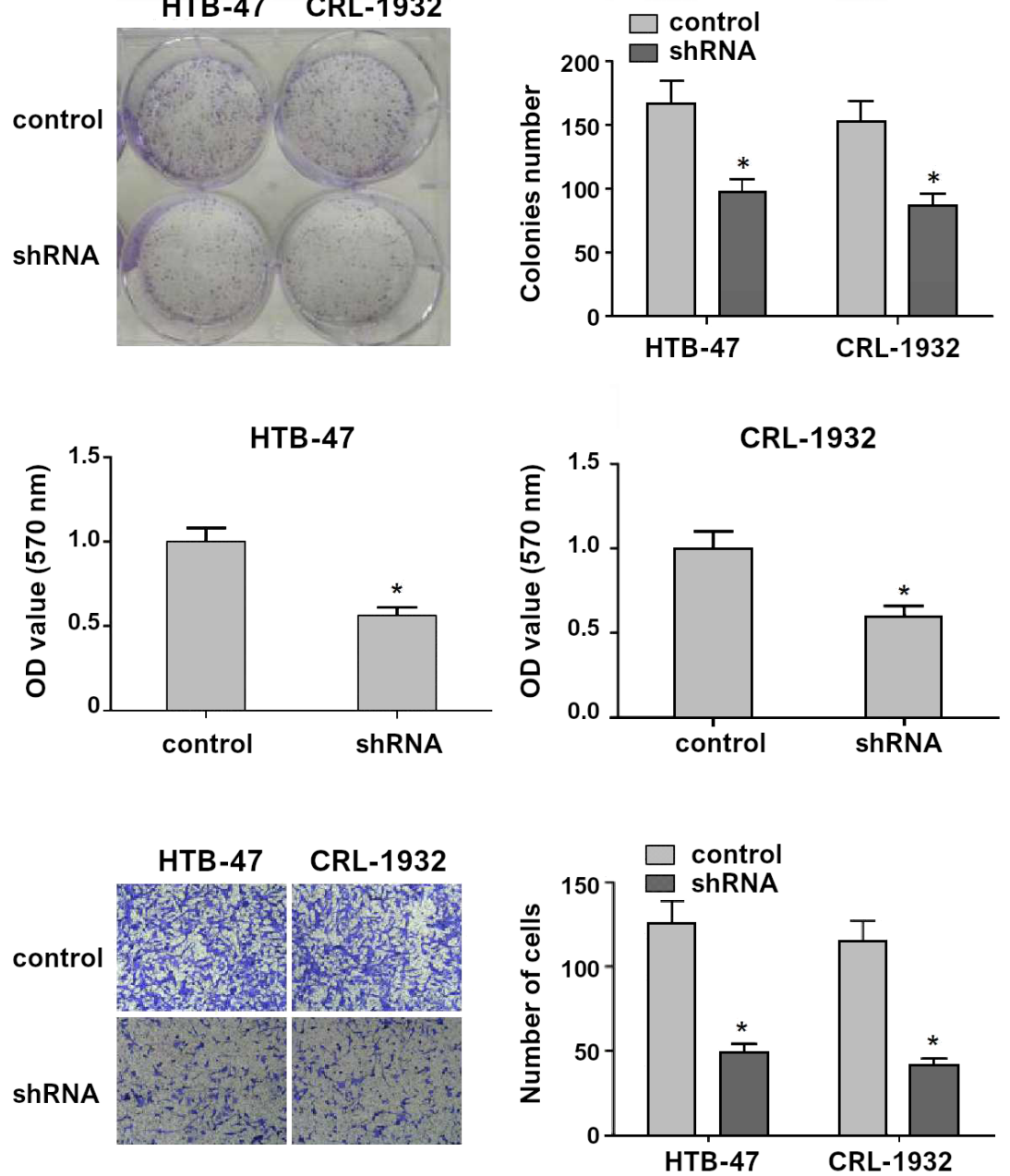

D
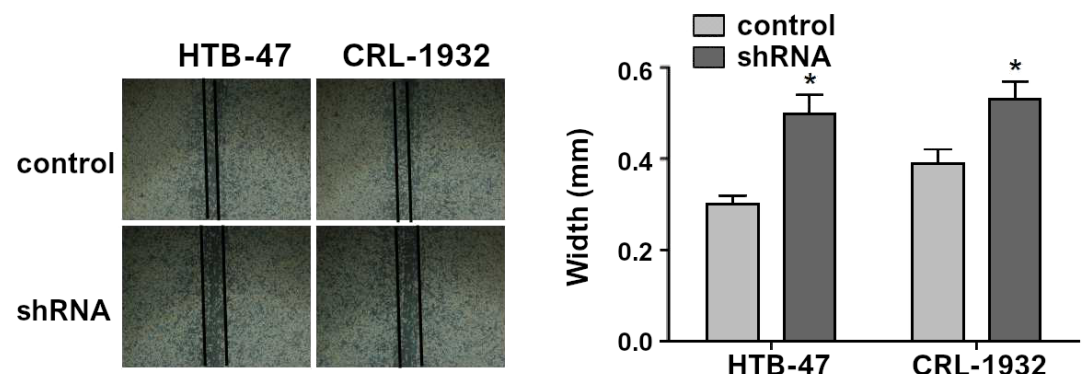
Collectively, we revealed that TTK contributes to the proliferation and invasion of ccRCC cells in vitro.

TTK promotes tumor growth and metastasis of ccRCC in mice. We further assessed the possibility that TTK contributes to tumor growth and metastasis of ccRCC in mice. To verify our expectation, HTB-47 cells were infected with control or TTK shRNA lentivirus and subcutaneously injected into nude mice. After 14 days, the volume of tumors was measured every week. Representative photographs are shown in Figure 4A. The growth curve was calculated, graphed and shown in Figure 4A. As we expected, the volume of tumors isolated from TTK ablation groups was obviously smaller than that in control (Figure 4A). Additionally, we performed lung metastasis assays in mice. HTB-47 cells were infected with TTK shRNA lentivirus to stably knockdown the expression of TTK. Subsequently, control or TTK ablation HTB-47 cells were injected into the caudal vein of nude mice. After 7 weeks, tumors were isolated from mice, photographed and the volume of tumors was measured. Similarly, we found that the incidence of lung metastasis for HTB-47 cells were significantly inhibited compared with control (Figure 4B). Meanwhile, the effective silencing of TTK in tumors isolated from mice was detected in TTK depletion groups (Figure 4C). According to these results, we demonstrated the tumor induction role of TTK in mice.

\section{Discussion}

In recent years, targeted therapy showed a promising prospect in the treatment of ccRCC $[20,21]$. In order to improve the prognosis of patients with ccRCC, more effective therapeutic targets are still needed. A number of studies confirmed the key role of TKK in the progression of multiple cancers, and various inhibitors targeting this protein have been developed $[14,22]$. However, the possible role of this protein in the development of ccRCC is still unclear. Here we found that TTK was highly expressed in human ccRCC tissues and promoted cell proliferation and invasion of ccRCC in vitro and in mice. Our results therefore confirmed the critical role of TTK in ccRCC development and suggested

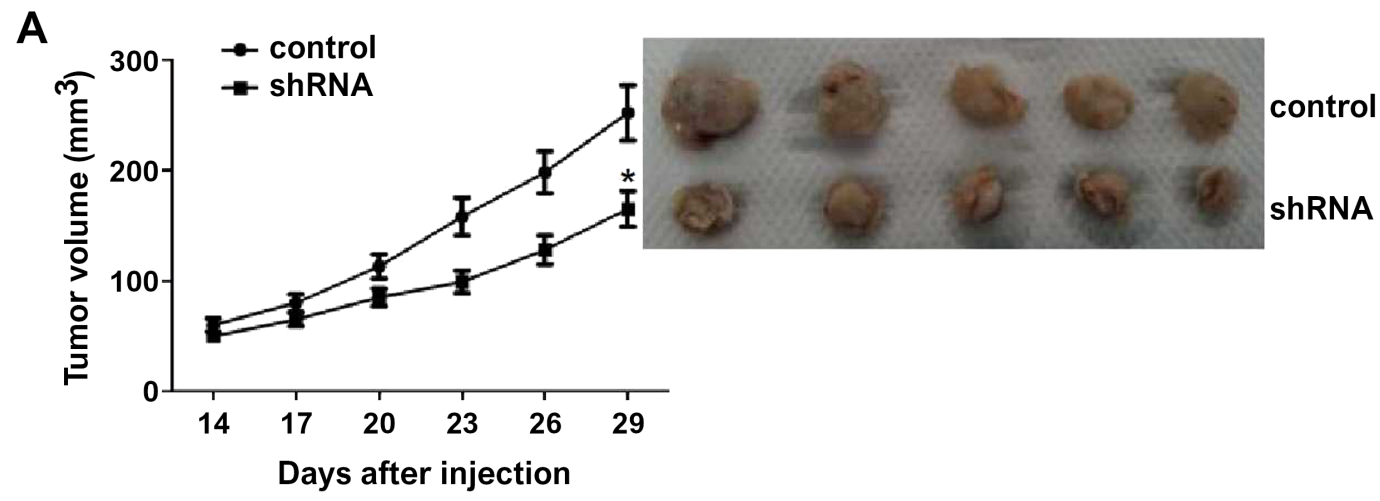

B

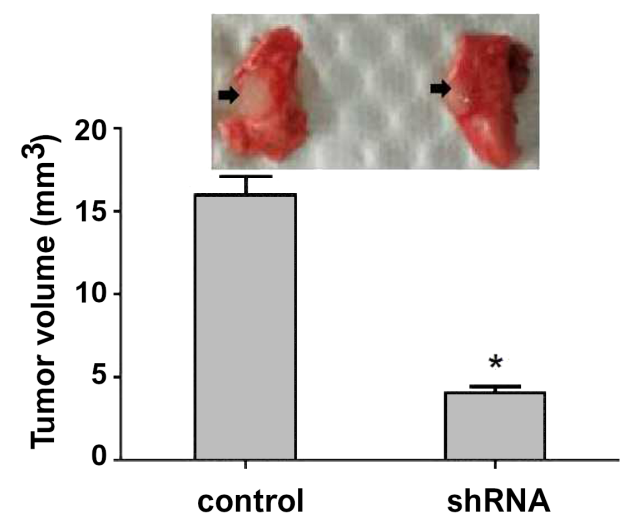

C

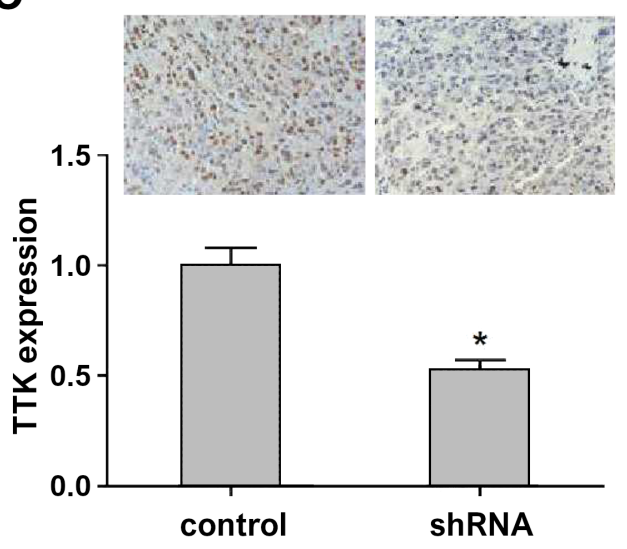

Figure 4. TTK promotes tumor growth and metastasis of ccRCC in mice. A) HTB-47 cells infected with control or TTK shRNA lentivirus were subcutaneously implanted into nude mice. Tumor growth curves were calculated and analyzed and evaluated according to volume of 5 tumors in TTK ablation and control groups. B) HTB-47 cells infected with control or TTK shRNA lentivirus were injected into the caudal vein of nude mice. After 8 weeks, tumors were isolated, photographed and weighted ( $\mathrm{n}=5$ for each group). C). IHC assays showed the expression levels of TTK in control or TTK depletion tumors isolated from mice. Results are presented as mean $\pm \mathrm{SD},{ }^{\star} \mathrm{p}<0.05$. 
that it could serve as a novel therapeutic target for the treatment of ccRCC.

In this study, we found the tumor-induction role of TTK in ccRCC. TTK is commonly involved in the regulation of proliferation, migration and invasion in multiple cancers $[23,24]$. TTK was demonstrated to be correlated with the prognosis of patients with glioma, and its overexpression was present in glioma and positively associates with tumor grade [25]. Interestingly, the inhibitors of TTK, such as Mps1-IN-3 and NMS-P715, have been developed to improve the prognosis of glioma, through enhancing cell death and inducing mitotic catastrophe [26, 27]. Additionally, TTK were significantly highly expressed in breast cancer cells and triple negative breast cancer (TNBC) samples, and obviously correlated with high histologic grade of patients [18]. TTK depletion could severely compromise the viability of TNBC cell lines and induced cell apoptosis [18]. A TTK inhibitor, NTRC0066-0, showed high potent in TNBC cells and mice model. In addition, TTK could also affect the development of lung cancer, esophageal cancer, hepatocellular carcinoma and pancreatic cancer $[17,28,29]$. Therefore, TTK had a potential to be used as biomarkers and molecular targets for these tumors [14]. The inhibitors are also under development, some of which have entered clinical trials [30]. We here found that TTK was highly expressed in ccRCC tissues and affected the proliferation and invasion of ccRCC cells, suggesting that TTK could therefore serve as a potential therapeutic target for the treatment of ccRCC. However, the precise molecular mechanism needs further study, and the inhibitors targeting TTK also need to be developed.

As we know, TTK is a key spindle assembly checkpoint kinase [31]. The overexpression of TTK was found in multiple cancers [31]. The depletion of TTK compromises spindle assembly checkpoint, leads to errors of chromosome missegregation and therefore blocks cancer cell survival and proliferation [11, 31]. We here found that TTK knockdown led to the inhibition of ccRCC cell proliferation, which might be caused by the inhibition of its spindle assembly checkpoint kinase activity. We next should detect the effects of TTK on cell cycle of ccRCC, and further investigate the regulatory mechanism underlying TTK promoting ccRCC proliferation.

To explore the possible link between TTK expression and ccRCC progression, the expression levels of TTK in 112 ccRCC tissues and the corresponding non-tumor tissues were detected through IHC assays. Compared with normal tissues, the expression levels of TTK in tumor tissues were obviously higher. The analysis on the link of clinical features exhibits that TTK expression levels were obviously associated with clinicopathological features including T stage $(\mathrm{p}=0.008)$ and lymphatic metastasis $(\mathrm{p}=0.023)$. T stage and lymphatic metastasis are related to tumor metastasis, which also implies that TTK affects the metastasis of ccRCC. We further performed transwell assays to confirm that TTK depletion inhibited the invasion of ccRCC cells and found that TTK contributed to the lung metastasis of ccRCC in mice. Our previous results also proved that TTK promoted ccRCC cell proliferation in vitro and tumor growth. TTK may therefore has multiple effects on the occurrence and development of ccRCC. We need to further clarify the relevant regulatory mechanism, so as to further understand the role of TTK in the development and metastasis of ccRCC and lay a foundation for the development of TTK-targeted inhibitors.

In conclusion, we found that TTK was highly expressed in human clear cell renal cell carcinoma tissues. We also investigated the correlation between TTK expression and clinical characteristics of patients who underwent ccRCC. Furthermore, TTK contributed to the proliferation and invasion capacity of ccRCC cells in vitro, and promoted tumor growth and metastasis of ccRCC in mice. We therefore mechanically confirmed that TTK promoted the development and metastasis of ccRCC, and provided TTK as a novel therapeutic molecular target for the treatment of ccRCC.

Supplementary information is available in the online version of the paper.

\section{References}

[1] BIE L, ZHAO G, CHENG P, RONDEAU G, PORWOLLIK $S$ et al. The accuracy of survival time prediction for patients with glioma is improved by measuring mitotic spindle checkpoint gene expression. PLoS One 2011; 6: e25631. https:// doi.org/10.1371/journal.pone.0025631

[2] MURPHY KA, JAMES BR, WILBER A, GRIFFITH TS. A Syngeneic Mouse Model of Metastatic Renal Cell Carcinoma for Quantitative and Longitudinal Assessment of Preclinical Therapies. J Vis Exp 2017; 55080. https://doi. org/10.3791/55080

[3] LANE BR, KATTAN MW. Predicting outcomes in renal cell carcinoma. Curr Opin Urol 2005; 15: 289-297. https://doi. org/10.1097/01.mou.0000178336.94991.17

[4] ALSHAREDI M, KATZ H. Check point inhibitors a new era in renal cell carcinoma treatment. Med Oncol 2018; 35: 85. https://doi.org/10.1007/s12032-018-1147-y

[5] WANG X, WANG T, CHEN C, WU Z, BAI P et al. Serum exosomal miR-210 as a potential biomarker for clear cell renal cell carcinoma. J Cell Biochem 2018. https://doi. org/10.1002/jcb. 27347

[6] GHATALIA P, ZIBELMAN MR, GEYNISMAN DM, PLIMACK ER. Evolving landscape of the treatment of metastatic clear cell renal cell carcinoma. Clin Adv Hematol Oncol 2018; 16: 677-686.

[7] SONG Y, HUANG J, SHAN L, ZHANG HT. Analyses of Potential Predictive Markers and Response to Targeted Therapy in Patients with Advanced Clear-cell Renal Cell Carcinoma. Chin Med J (Engl) 2015; 128: 2026-2033. https://doi. org/10.4103/0366-6999.161353

[8] RICKETTS CJ, LINEHAN WM. Insights into Epigenetic Remodeling in VHL-Deficient Clear Cell Renal Cell Carcinoma. Cancer Discov 2017; 7: 1221-1223. https://doi. org/10.1158/2159-8290.CD-17-0971 
[9] HOGNER A, KRAUSE H, JANDRIG B, KASIM M, FULLER TF et al. PBRM1 and VHL expression correlate in human clear cell renal cell carcinoma with differential association with patient's overall survival. Urol Oncol 2018; 36: 94. https://doi.org/10.1016/j.urolonc.2017.10.027

[10] RIGGS JR, NAGY M, ELSNER J, ERDMAN P, CASHION $D$ et al. The Discovery of a Dual TTK Protein Kinase/CDC2Like Kinase (CLK2) Inhibitor for the Treatment of Triple Negative Breast Cancer Initiated from a Phenotypic Screen. J Med Chem 2017; 60: 8989-9002. https://doi.org/10.1021/ acs.jmedchem.7b01223

[11] PACHIS ST, KOPS G. Leader of the SAC: molecular mechanisms of Mps1/TTK regulation in mitosis. Open Biol 2018, 8. https://doi.org/10.1098/rsob.180109

[12] WEI JH, CHOU YF, OU YH, YEH YH, TYAN SW et al. TTK/hMps 1 participates in the regulation of DNA damage checkpoint response by phosphorylating CHK2 on threonine 68. J Biol Chem 2005; 280: 7748-7757. https://doi. org/10.1074/jbc.M410152200

[13] NITTYMAKI I, GYLFE A, LAINE L, LAAKSO M, LEHTONEN HJ et al. High frequency of TTK mutations in microsatellite-unstable colorectal cancer and evaluation of their effect on spindle assembly checkpoint. Carcinogenesis 2011; 32: 305-311. https://doi.org/10.1093/carcin/bgq272

[14] SUGIMOTO Y, SAWANT DB, FISK HA, MAO L, LI C et al. Novel pyrrolopyrimidines as Mps1/TTK kinase inhibitors for breast cancer. Bioorg Med Chem 2017; 25: 2156-2166. https://doi.org/10.1016/j.bmc.2017.02.030

[15] AHN CH, KIM YR, KIM SS, YOO NJ, LEE SH. Mutational analysis of TTK gene in gastric and colorectal cancers with microsatellite instability. Cancer Res Treat 2009; 41: 224228. https://doi.org/10.4143/crt.2009.41.4.224

[16] SALVATORE G, NAPPI TC, SALERNO P, JIANG Y, GARBI $\mathrm{C}$ et al. A cell proliferation and chromosomal instability signature in anaplastic thyroid carcinoma. Cancer Res 2007; 67: 10148-10158. https://doi.org/10.1158/0008-5472.CAN-07-1887

[17] CHEN X, YU C, GAO J, ZHU H, CUI B et al. A novel USP9X substrate TTK contributes to tumorigenesis in nonsmall-cell lung cancer. Theranostics 2018; 8: 2348-2360. https://doi.org/10.7150/thno.22901

[18] MAIRE V, BALDEYRON C, RICHARDSON M, TESSON B, VINCENT-SALOMON A, GRAVIER E et al. TTK/hMPS1 is an attractive therapeutic target for triple-negative breast cancer. PLoS One 2013; 8: e63712. https://doi.org/10.1371/ journal.pone.006371

[19] LIU Y, LANG Y, PATEL NK, NG G, LAUFER R et al. The Discovery of Orally Bioavailable Tyrosine Threonine Kinase (TTK) Inhibitors: 3-(4-(heterocyclyl)phenyl)-1H-indazole5-carboxamides as Anticancer Agents. J Med Chem 2015; 58: 3366-3392. https://doi.org/10.1021/jm501740a

[20] ITO K, MASUNAGA A, TANAKA N, MIZUNO R, SHIROTAKE $S$ et al. Impact of inflammatory marker levels one month after the first-line targeted therapy initiation on progression-free survival prediction in patients with metastatic clear cell renal cell carcinoma. Jpn J Clin Oncol 2019; 49: 69-76. https://doi.org/10.1093/jjco/hyy154
[21] SACRE A, BARTHELEMY P, KORENBAUM C, BURGY $\mathrm{M}$, WOLTER P et al. Prognostic factors in second-line targeted therapy for metastatic clear-cell renal cell carcinoma after progression on an anti-vascular endothelial growth factor receptor tyrosine kinase inhibitor. Acta Oncol 2016; 55: 329-340. https://doi.org/10.3109/0284186X.2015.1099731

[22] ZAMAN GJR, DE ROOS JADM, LIBOUBAN MAA, PRINSEN MBW, DE MAN J et al. TTK Inhibitors as a Targeted Therapy for CTNNB1 (beta-catenin) Mutant Cancers. Mol Cancer Ther 2017; 16: 2609-2617. https://doi. org/10.1158/1535-7163.MCT-17-0342

[23] KING JL, ZHANG B, LI Y, LI KP, NI JJ et al. TTK promotes mesenchymal signaling via multiple mechanisms in triple negative breast cancer. Oncogenesis 2018; 7: 69. https://doi. org/10.1038/s41389-018-0077-z

[24] STRATFORD JK, YAN F, HILL RA, MAJOR MB, GRAVES LM et al. Genetic and pharmacological inhibition of TTK impairs pancreatic cancer cell line growth by inducing lethal chromosomal instability. PLoS One 2017; 12: e0174863. https://doi.org/10.1371/journal.pone.0174863

[25] KUSAKABE K, IDE N, DAIGO Y, ITOH T, YAMAMOTO $\mathrm{T}$ et al. A unique hinge binder of extremely selective aminopyridine-based Mps1 (TTK) kinase inhibitors with cellular activity. Bioorg Med Chem 2015; 23: 2247-2260. https://doi. org/10.1016/j.bmc.2015.02.042

[26] TANNOUS BA, KERAMI M, VAN DER STOOP PM, KWIATKOWSKI N, WANG J et al. Effects of the selective MPS1 inhibitor MPS1-IN-3 on glioblastoma sensitivity to antimitotic drugs. J Natl Cancer Inst 2013; 105: 1322-1331. https:// doi.org/10.1093/jnci/djt168

[27] COLOMBO R, CALDARELLI M, MENNECOZZI M, GIORGINI ML, SOLA F et al. Targeting the mitotic checkpoint for cancer therapy with NMS-P715, an inhibitor of MPS1 kinase. Cancer Res 2010; 70: 10255-10264. https:// doi.org/10.1158/0008-5472.CAN-10-2101

[28] LIANG XD, DAI YC, LI ZY, GAN MF, ZHANG SR et al. Expression and function analysis of mitotic checkpoint genes identifies TTK as a potential therapeutic target for human hepatocellular carcinoma. PLoS One 2014; 9: e97739. https:// doi.org/10.1371/journal.pone.0097739

[29] KAISTHA BP, HONSTEIN T, MULLER V, BIELAK S, SAUER $M$ et al. Key role of dual specificity kinase TTK in proliferation and survival of pancreatic cancer cells. Br J Cancer 2014; 111: 1780-1787. https://doi.org/10.1038/bjc.2014.460

[30] MASON JM, WEI X, FLETCHER GC, KIARASH R, BROKX R et al. Functional characterization of CFI-402257, a potent and selective Mps1/TTK kinase inhibitor, for the treatment of cancer. Proc Natl Acad Sci U S A 2017; 114: 3127-3132. https://doi.org/10.1073/pnas.1700234114

[31] XIE Y, WANG A, LIN J, WU L, ZHANG H et al. Mps1/ TTK: a novel target and biomarker for cancer. J Drug Target 2017; 25: 112-118. https://doi.org/10.1080/106118 6X.2016.1258568 